\title{
Machine Learning Derived Blueprint for Rational Design of the Effective Single-Atom Cathode Catalyst of Lithium-Sulfur Battery
}

Zan Lian, ${ }^{1,2}$ Min Yang, ${ }^{1,2}$ Faheem Jan, ${ }^{1,2}$ and Bo $\mathrm{Li}^{*}{ }^{* 1,2}$

${ }^{1}$ Shenyang National Laboratory for Materials Science, Institute of Metal Research, Chinese Academy of Sciences, Shenyang 110016, Liaoning, People's Republic of China

${ }^{2}$ School of Materials Science and Engineering, University of Science and Technology of China, Shenyang 110016, Liaoning, People's Republic of China

*email: boli@imr.ac.cn 


\section{Computational Methods}

\section{DFT calculation}

The first principle calculations reported in this work are performed using periodic, spin-polarized density functional theory, which is implemented in Vienna ab initio simulation package (VASP). ${ }^{1-3}$ For valence electrons, a plane-wave basis set is adopted with an energy cutoff of $400 \mathrm{eV}$ and the ionic cores are described by the projector augmented-wave (PAW) method. ${ }^{4-5}$ RPBE functional is employed as the exchange-correlation functional approximation. ${ }^{6}$ Moreover, DFT-D3 correction method of Grimme et al. is used to calculate the van der Waals correction. ${ }^{7}$ A $3 \times 3 \times 1$ Monk horstPack k-point grid is used to sample the Brillion zone. The total energy was converged to an accuracy of $1 \times 10^{-6} \mathrm{eV}$ to obtain accurate forces, and a force tolerance of $0.03 \mathrm{eV} / \AA$ was used in all structure optimization. $3 \mathrm{~d}, 4 \mathrm{~d}$, and $5 \mathrm{~d}$ single metal atom anchoring on seven doped graphene model are considered as shown in Figure $\mathrm{S} 1$. $\mathrm{Li}_{2} \mathrm{~S}, \mathrm{Li}_{2} \mathrm{~S}_{2}, \mathrm{Li}_{2} \mathrm{~S}_{4}$, and $\mathrm{S}_{8}$ are considered as typical polysulfides in Li-S battery. The initial adsorption configurations of $\mathrm{Li}_{2} \mathrm{~S}, \mathrm{Li}_{2} \mathrm{~S}_{2}$, and $\mathrm{S}_{8}$ are enumerated by considering all equivalent sites and molecular symmetry. For $\mathrm{Li}_{2} \mathrm{~S}_{4}$, the complexity is significantly increased. The most stable configurations of $\mathrm{Li}_{2} \mathrm{~S}_{4}$ in gas phase and adsorption state are identified by using the minima hopping algorithm as implemented in Atomic Simulation Environment (ASE). ${ }^{8}$ The electronic structure analysis are performed via bader charge analysis and DDEC6 atomic population analysis. ${ }^{9-10}$ The judgment of bonding are performed by data set of Open Babel implemented in Jmol. ${ }^{11}$

\section{Modification of CGCNN and machine learning}

The modified framework of previous reported $\mathrm{CGCNN}^{12}$ is used in current work. The original CGCNN method represented a crystal structure by a graph, which encodes the information of atoms and bonds by corresponding atom feature vector $v_{i}$ and bond feature vector $u_{(i, j) k}$. Then, a convolutional neural network (CNN) is built based on the crystal graphs and target properties. The major goal of current work is to estimate the binding energies of LiPS which involved two separate units, catalyst and polysulfides. Therefore, these two separate parts are unified to a graph (Figure S2) which is accomplished by appending the two feature vectors of polysulfides to vectors of catalyst for each sample. The mathematical form of the graphs is $G_{C G C N N}=\left(\left\{v_{c 1}, v_{c 2}, v_{c 3}, \ldots\right\},\left\{u_{c 1}\right.\right.$, $\left.\left.c 2, u_{c 2, c 3}, \ldots\right\}\right), G_{m o d i f i e d}=\left(\left\{v_{s 1}, v_{s 2}, v_{s 3}, \ldots, v_{m 1}, v_{m 2}, v_{m 3}, \ldots,\right\},\left\{u_{s 1, s 2}, u_{s 2}, s 3, \ldots, u_{m 1, m 2}, u_{m 2}, m 3, \ldots\right\}\right)$. Where $G_{C G C N N}$ and $G_{\text {modified }}$ represents the graph built from CGCNN and modified CGCNN frame. $v_{c i}$ and $u_{c i, c j}$ represent feature vector of atom $\mathrm{i}$ and bond between atom $\mathrm{i}$ and $\mathrm{j}$ in crystal. $v_{s i}$ and $u_{s i}$, ${ }_{s j}$ represent feature vector of atom $\mathrm{i}$ and bond between atom $\mathrm{i}$ and $\mathrm{j}$ in slab. $v_{m i}$ and $u_{m i, m j}$ represent feature vector of atom $i$ and bond between atom $i$ and $j$ in molecular. The obtained graph was used in the following CNN process ${ }^{12}$. The data set from DFT calculations is randomly divided into three sets, training set (60\%), validation set (20\%), and test set $(20 \%)$ for regression and classification. In addition, the Imbalanced Dataset Sampler ${ }^{13}$ was applied in training.

\section{Overpotential calculation}

The model and elementary steps used for overpotential calculation are shown below which is inspired by the computational hydrogen electrode approach in ORR. ${ }^{14-15}$

$$
\begin{gathered}
\mathrm{Li}_{2} \mathrm{~S}_{2}+\mathrm{Li}^{+}+\mathrm{e}^{-}+* \rightarrow \mathrm{LiS}^{*}+\mathrm{Li}_{2} \mathrm{~S} \\
\mathrm{LiS}^{*}+\mathrm{Li}^{+}+\mathrm{e}^{-} \rightarrow *+\mathrm{Li}_{2} \mathrm{~S}
\end{gathered}
$$


The computational equilibrium potential was calculated by:

$$
\mathrm{U}_{\text {eq }}=-\left(2 \mathrm{G}\left(\mathrm{Li}_{2} \mathrm{~S}\right)-2 \mathrm{G}(\mathrm{Li})-\mathrm{G}\left(\mathrm{Li}_{2} \mathrm{~S}_{2}\right)\right) / 2 \mathrm{e}=2.147 \mathrm{~V}
$$

The free energy of $\mathrm{Li}^{+}$and $\mathrm{e}^{-}$at $\mathrm{U}=0$ relative to the $\mathrm{Li} / \mathrm{Li}^{+}$electrode:

$$
\mathrm{G}\left(\mathrm{Li}^{+}\right)+\mathrm{G}\left(\mathrm{e}^{-}\right)=\mathrm{G}(\mathrm{Li})
$$

The free energy change of the two reactions is calculated by:

$$
\begin{gathered}
\Delta \mathrm{G}\left(\mathrm{LiS}^{*}\right)=\mathrm{G}\left(\mathrm{LiS}^{*}\right)-\mathrm{G}\left({ }^{*}\right)-\mathrm{G}(\mathrm{Li})-\mathrm{G}\left(\mathrm{Li}_{2} \mathrm{~S}_{2}\right)+\mathrm{G}\left(\mathrm{Li}_{2} \mathrm{~S}\right) \\
=\mathrm{E}_{\mathrm{ad}}\left(\mathrm{LiS}^{*}\right)+\mathrm{G}(\mathrm{LiS})+\mathrm{G}\left(^{*}\right)-\mathrm{G}(*)-\mathrm{G}(\mathrm{Li})-\mathrm{G}\left(\mathrm{Li}_{2} \mathrm{~S}_{2}\right)+\mathrm{G}\left(\mathrm{Li}_{2} \mathrm{~S}\right) \\
=\mathrm{E}_{\mathrm{ad}}\left(\mathrm{LiS} \mathrm{S}^{*}\right)+\mathrm{G}(\mathrm{LiS})-\mathrm{G}(\mathrm{Li})-\mathrm{G}\left(\mathrm{Li}_{2} \mathrm{~S}_{2}\right)+\mathrm{G}\left(\mathrm{Li}_{2} \mathrm{~S}\right) \\
\triangle \mathrm{G}_{1}=\Delta \mathrm{G}\left(\mathrm{LiS}^{*}\right) \\
\Delta \mathrm{G}_{2}=-\Delta \mathrm{G}\left(\mathrm{LiS}^{*}\right)+2 \mathrm{G}\left(\mathrm{Li}_{2} \mathrm{~S}\right)-2 \mathrm{G}(\mathrm{Li})-\mathrm{G}\left(\mathrm{Li}_{2} \mathrm{~S}_{2}\right)
\end{gathered}
$$

The limiting potential of step $i$ and overpotential of the overall reaction are:

$$
\begin{gathered}
U_{\mathrm{L}, \mathrm{i}}=-\Delta \mathrm{G}_{\mathrm{i}} / \mathrm{e} \\
\eta=\mathrm{U}_{\text {eq }}-\min \left(\mathrm{U}_{\mathrm{L}, \mathrm{i}}\right)
\end{gathered}
$$

Where, $\mathrm{G}=\mathrm{E}_{\mathrm{DFT}}+\mathrm{ZPE}-\mathrm{TS}$. $\mathrm{Li}, \mathrm{Li}_{2} \mathrm{~S}$, and $\mathrm{Li}_{2} \mathrm{~S}_{2}$ are in solid state and $\mathrm{LiS}$ in gas phase. $\triangle \mathrm{G}\left(\mathrm{LiS}^{*}\right)$ is the adsorption energy of $\mathrm{LiS}$ relative to *, $\mathrm{Li}_{1} \mathrm{Li}_{2} \mathrm{~S}$, and $\mathrm{Li}_{2} \mathrm{~S}_{2}$ solid. $\mathrm{E}_{\mathrm{ad}}\left(\mathrm{LiS}^{*}\right)$ is the adsorption energy of LiS relative to * and LiS gas. Considering the low contribution of the correction of ZPE - $\mathrm{TS}^{14}$, It is approximated to be zero in this work. 

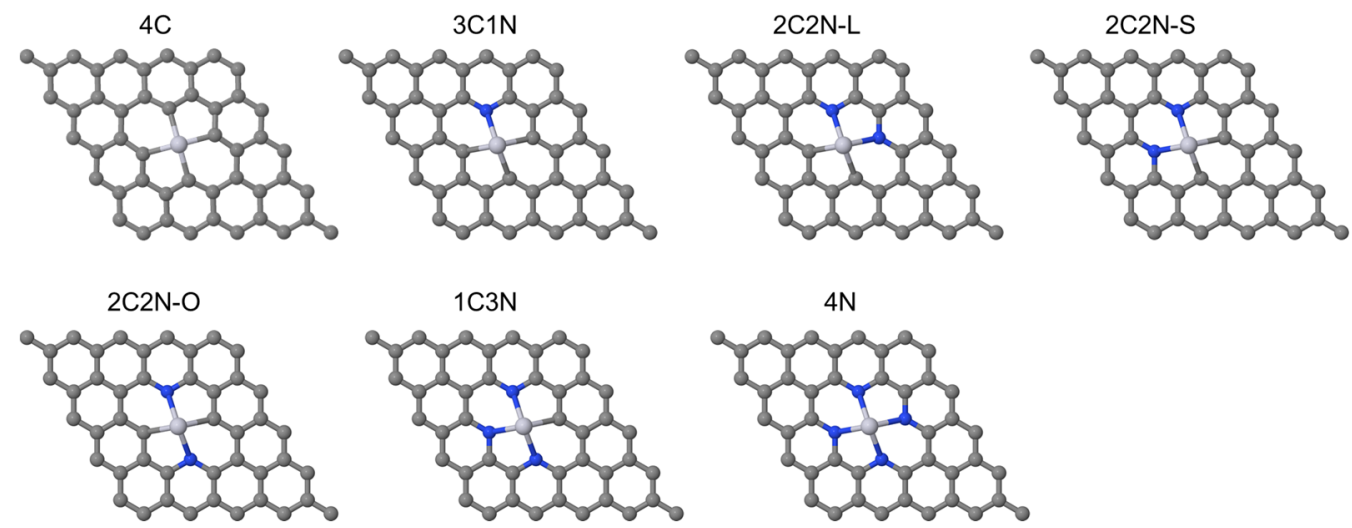

Figure S1. The configurations of seven types of SACs. C in dark gray, $\mathrm{N}$ in blue, and metal in light gray.

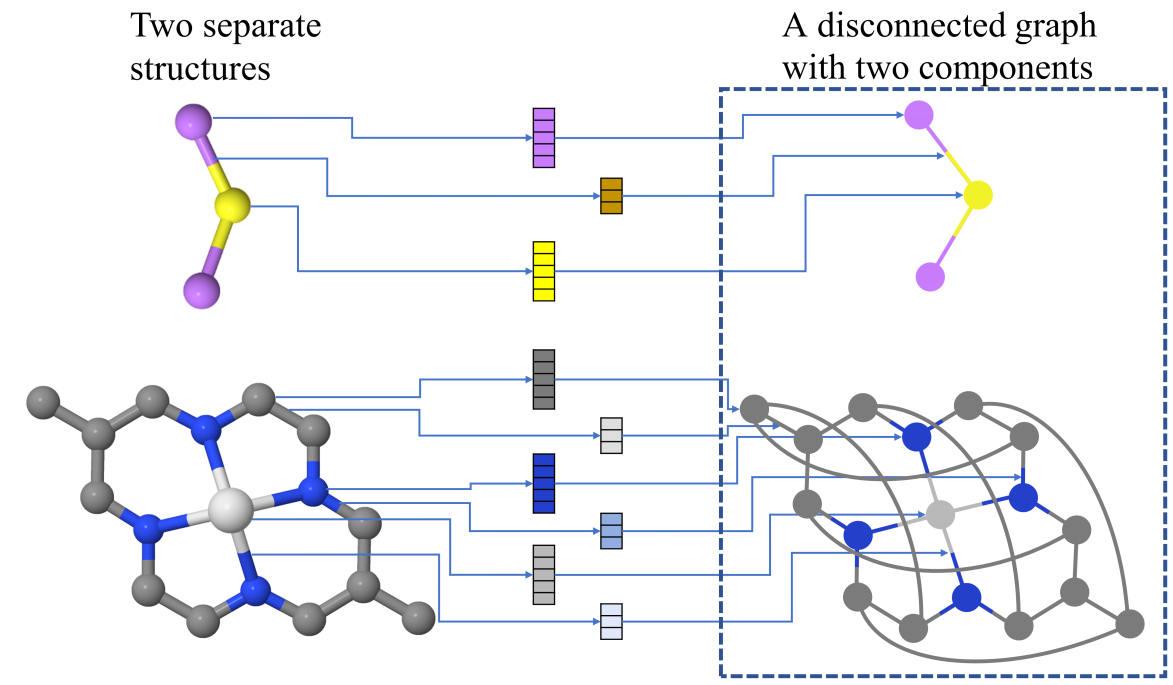

Figure S2. Construction of the graph in modified CGCNN.
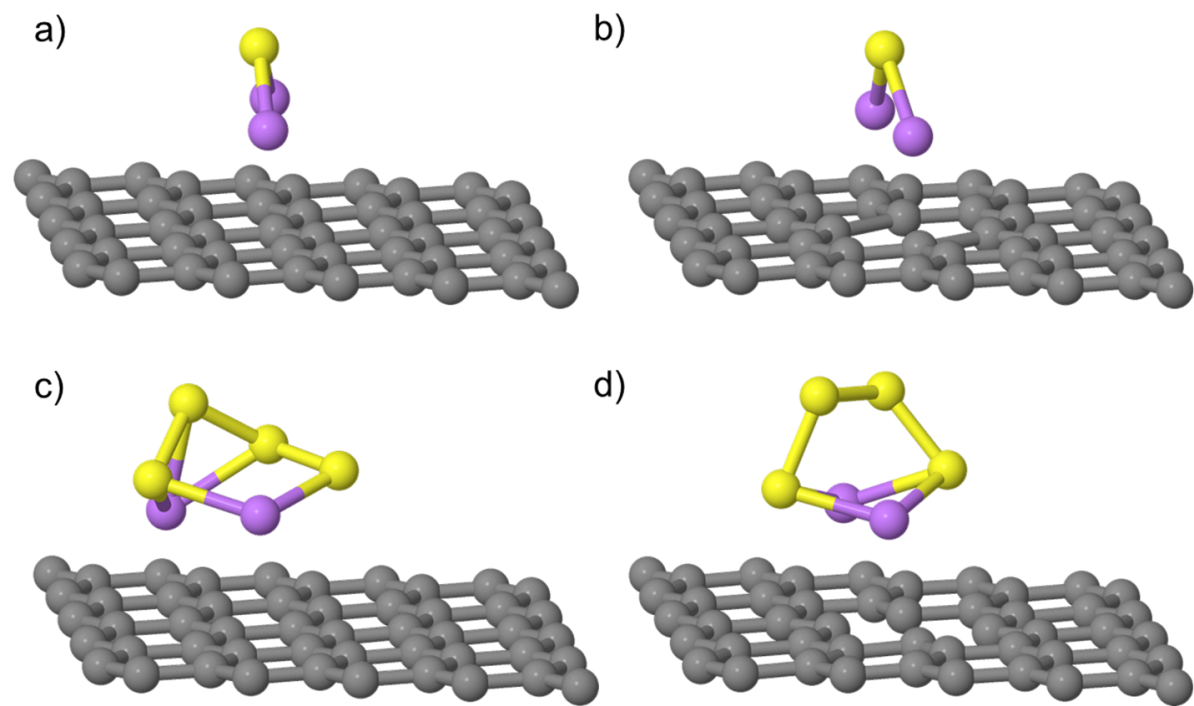

d)

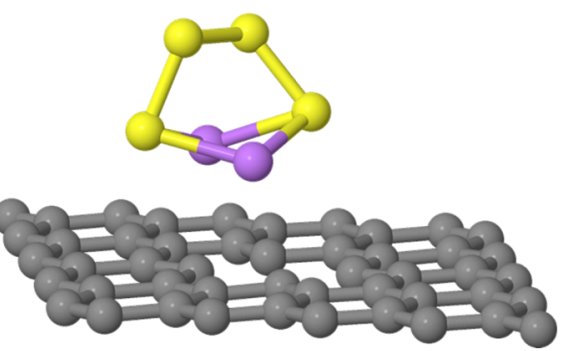

Figure $\mathrm{S} 3$. The adsorption geometry of $\mathrm{Li}_{2} \mathrm{~S}$ and $\mathrm{Li}_{2} \mathrm{~S}_{4}$ on pristine and defective graphene. a) $\mathrm{Li}_{2} \mathrm{~S}$ on pristine graphene, b) $\mathrm{Li}_{2} \mathrm{~S}$ on defective graphene, c) $\mathrm{Li}_{2} \mathrm{~S}_{4}$ on pristine graphene, and d) $\mathrm{Li}_{2} \mathrm{~S}_{4}$ on defective graphene. 


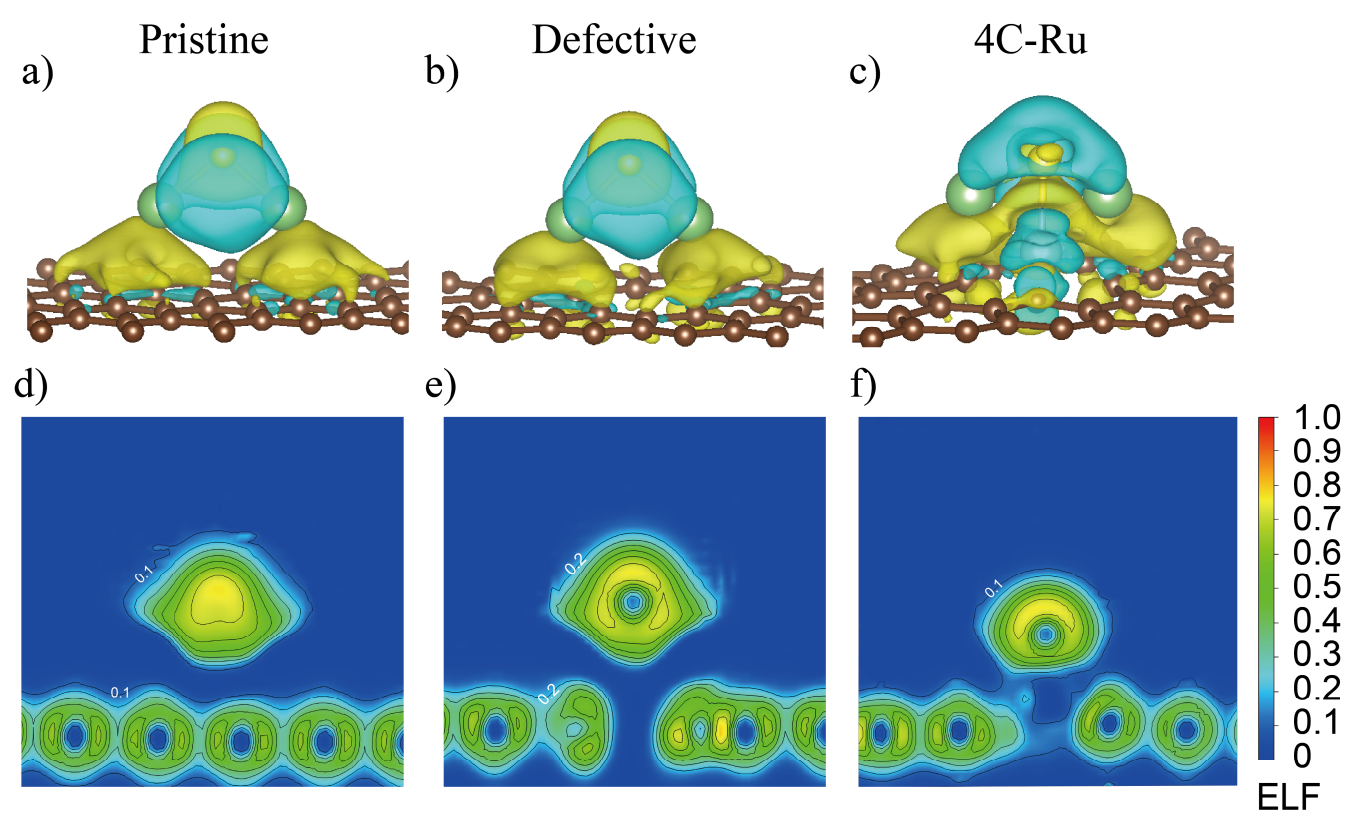

Figure S4. The electronic structure of $\mathrm{Li}_{2} \mathrm{~S}$ adsorbed on three catalysts. Isosurface of charge density difference (a-c), and two-dimensional cross-section view of charge density difference (d-f) on pristine graphene, defective graphene, and $4 \mathrm{C}-\mathrm{Ru}$. The cyan represents decreased charge density, and yellow represents increased charge density in (a-c). The step-size of contour line is 0.1 in (d-f).
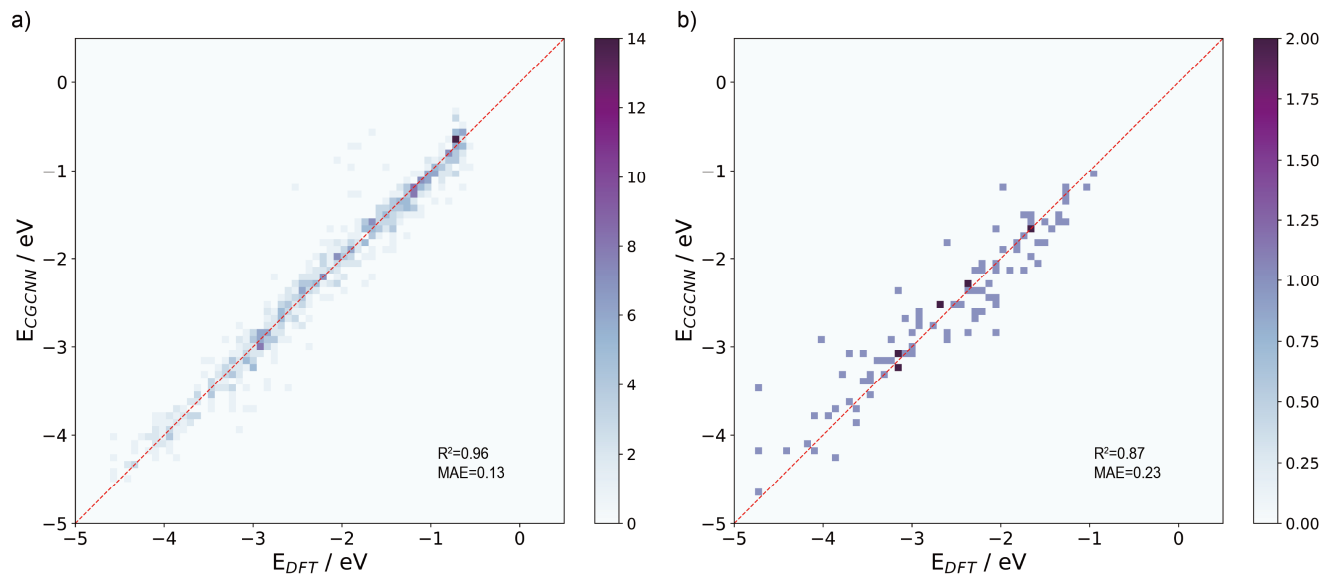

Figure S5. Two dimensional histograms of DFT calculated and ML predicted adsorption energy of LiPSs in category of a) $\mathrm{C} 1 / \mathrm{C} 2$ and b) $\mathrm{C} 3$. The color scale is used to illustrate the magnitude of number in samples. 


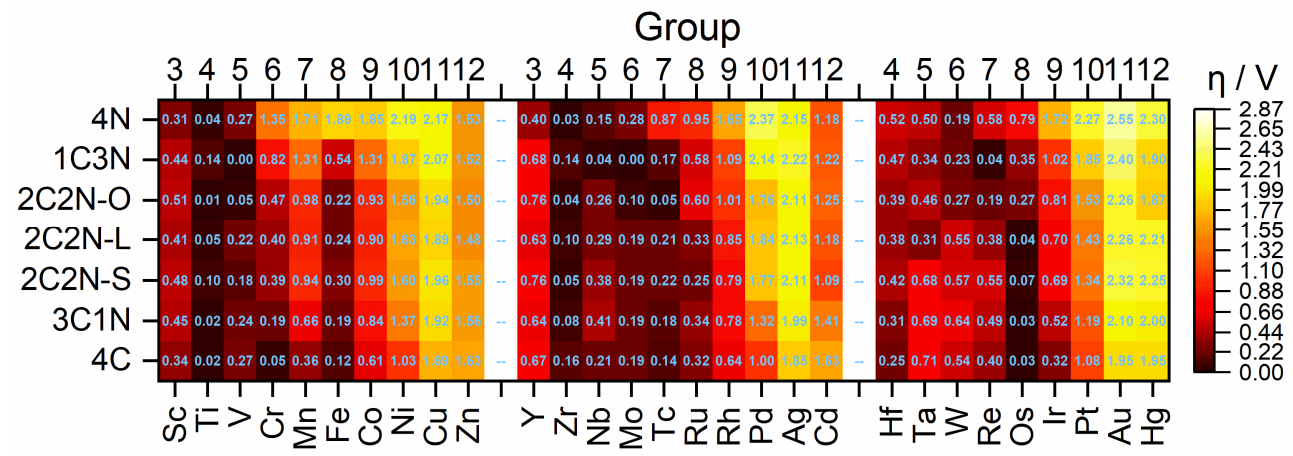

Figure S6. The heat map of predicted overpotential of different SACs. The number in the block is the overpotential. The value is 0.003 for $1 \mathrm{C} 3 \mathrm{~N}-\mathrm{V}$ and $1 \mathrm{C} 3 \mathrm{~N}-\mathrm{Mo}$.

Table $\mathrm{S} 1$. The net charge of isolated $\mathrm{Li}_{2} \mathrm{~S}$ and adsorbed $\mathrm{Li}_{2} \mathrm{~S}$ on three catalysts.

\begin{tabular}{ccccccccc}
\hline & \multicolumn{4}{c}{ DDEC6 net charge } & \multicolumn{3}{c}{ Bader net charge } \\
\hline Element & Gas & Pristine & Defective & 4C-Ru & Gas & Pristine & Defective & 4C-Ru \\
\hline $\mathbf{S}$ & -1.7 & -1.25 & -1.04 & -0.87 & -1.66 & -1.3 & -1.11 & -0.96 \\
\hline $\mathbf{L i}$ & 0.85 & 0.80 & 0.76 & 0.80 & 0.87 & 0.87 & 0.87 & 0.87 \\
\hline $\mathbf{L i}$ & 0.85 & 0.81 & 0.77 & 0.79 & 0.87 & 0.88 & 0.87 & 0.87 \\
\hline Total & 0 & 0.35 & 0.50 & 0.71 & 0.08 & 0.45 & 0.63 & 0.79 \\
\hline
\end{tabular}

Table S2. The bond order of different bonding in isolated $\mathrm{Li}_{2} \mathrm{~S}$ and adsorbed $\mathrm{Li}_{2} \mathrm{~S}$.

\begin{tabular}{ccccc}
\hline \multicolumn{5}{c}{ DDEC6 bond order } \\
\hline bond & Gas & Pristine & Defective & 4 C-Ru \\
\hline $\mathbf{L i}-\mathbf{S}$ & 0.42 & 0.34 & 0.32 & 0.18 \\
\hline $\mathbf{L i}-\mathbf{S}$ & 0.42 & 0.33 & 0.32 & 0.19 \\
\hline $\mathbf{L i} / \mathbf{S}-\mathbf{C}$ & - & $<0.05$ & $<0.09$ & $<0.07$ \\
\hline $\mathbf{S}-\mathbf{R u}$ & - & - & - & 1.25 \\
\hline
\end{tabular}

Table S3. The specificity and recall in different sets under a threshold of 0.5.

\begin{tabular}{ccc}
\hline & Specificity & Recall \\
\hline Training set & 0.83 & 1.0 \\
\hline Validation set & 0.74 & 1.0 \\
\hline Test set & 0.79 & 1.0 \\
\hline
\end{tabular}


Table S4. Predicted adsorption energy of LiS.

\begin{tabular}{|c|c|c|c|c|c|c|c|}
\hline & $4 \mathrm{C}$ & $3 \mathrm{C} 1 \mathrm{~N}$ & $2 \mathrm{C} 2 \mathrm{~N}-\mathrm{S}$ & $2 \mathrm{C} 2 \mathrm{~N}-\mathrm{L}$ & $2 \mathrm{C} 2 \mathrm{~N}-\mathrm{O}$ & $1 \mathrm{C} 3 \mathrm{~N}$ & $4 N$ \\
\hline Sc & -3.28 & -3.17 & -3.13 & -3.2 & -3.11 & -3.17 & -3.3 \\
\hline $\mathbf{T i}$ & -3.6 & -3.64 & -3.72 & -3.66 & -3.62 & -3.76 & -3.65 \\
\hline $\mathbf{V}$ & -3.89 & -3.85 & -3.79 & -3.84 & -3.67 & -3.61 & -3.35 \\
\hline $\mathrm{Cr}$ & -3.56 & -3.42 & -3.23 & -3.22 & -3.15 & -2.8 & -2.26 \\
\hline Mn & -3.25 & -2.96 & -2.68 & -2.7 & -2.63 & -2.31 & -1.91 \\
\hline $\mathrm{Fe}$ & -3.5 & -3.43 & -3.32 & -3.38 & -3.39 & -3.08 & -1.72 \\
\hline Co & -3 & -2.77 & -2.63 & -2.71 & -2.69 & -2.3 & -1.77 \\
\hline $\mathbf{N i}$ & -2.59 & -2.24 & -2.02 & -1.99 & -2.05 & -1.75 & -1.42 \\
\hline $\mathrm{Cu}$ & -1.92 & -1.7 & -1.66 & -1.72 & -1.68 & -1.55 & -1.45 \\
\hline $\mathbf{Z n}$ & -1.98 & -2.06 & -2.07 & -2.14 & -2.11 & -2.1 & -2.09 \\
\hline $\mathbf{Y}$ & -2.94 & -2.97 & -2.86 & -2.99 & -2.86 & -2.94 & -3.21 \\
\hline $\mathbf{Z r}$ & -3.45 & -3.54 & -3.67 & -3.71 & -3.65 & -3.76 & -3.64 \\
\hline $\mathbf{N b}$ & -3.82 & -4.02 & -3.99 & -3.9 & -3.87 & -3.66 & -3.77 \\
\hline Mo & -3.81 & -3.8 & -3.81 & -3.8 & -3.71 & -3.61 & -3.34 \\
\hline Tc & -3.75 & -3.79 & -3.83 & -3.83 & -3.56 & -3.45 & -2.74 \\
\hline $\mathbf{R u}$ & -3.3 & -3.27 & -3.37 & -3.28 & -3.02 & -3.03 & -2.66 \\
\hline $\mathbf{R h}$ & -2.97 & -2.83 & -2.82 & -2.76 & -2.61 & -2.53 & -1.97 \\
\hline $\mathbf{P d}$ & -2.61 & -2.29 & -1.84 & -1.78 & -1.85 & -1.48 & -1.24 \\
\hline Ag & -1.76 & -1.62 & -1.5 & -1.48 & -1.51 & -1.4 & -1.47 \\
\hline Cd & -1.99 & -2.2 & -2.52 & -2.44 & -2.36 & -2.39 & -2.43 \\
\hline Hf & -3.87 & -3.93 & -4.03 & -3.99 & -4 & -4.08 & -4.13 \\
\hline Ta & -4.32 & -4.3 & -4.3 & -3.93 & -4.08 & -3.95 & -4.11 \\
\hline $\mathbf{W}$ & -4.15 & -4.25 & -4.19 & -4.17 & -3.89 & -3.84 & -3.43 \\
\hline $\mathbf{R e}$ & -4.02 & -4.1 & -4.17 & -4 & -3.8 & -3.65 & -3.04 \\
\hline Os & -3.65 & -3.65 & -3.68 & -3.58 & -3.35 & -3.26 & -2.82 \\
\hline $\mathbf{I r}$ & -3.29 & -3.1 & -2.92 & -2.91 & -2.8 & -2.59 & -1.9 \\
\hline $\mathbf{P t}$ & -2.54 & -2.43 & -2.27 & -2.19 & -2.09 & -1.77 & -1.35 \\
\hline $\mathbf{A u}$ & -1.67 & -1.51 & -1.3 & -1.36 & -1.36 & -1.22 & -1.07 \\
\hline $\mathbf{H g}$ & -1.67 & -1.61 & -1.37 & -1.4 & -1.74 & -1.71 & -1.32 \\
\hline
\end{tabular}


Table S5. Predicted adsorption energy of $\mathrm{Li}_{2} \mathrm{~S}_{3}$.

\begin{tabular}{cccccccc}
\hline & $\mathbf{4 C}$ & $\mathbf{3 C 1 N}$ & $\mathbf{2 C 2 N}-\mathbf{S}$ & $\mathbf{2 C 2 N}-\mathbf{L}$ & $\mathbf{2 C 2 N}-\mathbf{O}$ & $\mathbf{1 C 3 N}$ & $\mathbf{4 N}$ \\
\hline $\mathbf{S c}$ & -2.04 & -1.97 & -2.01 & -2.07 & -1.98 & -2.19 & -2.61 \\
$\mathbf{T i}$ & -2.15 & -2.24 & -2.53 & -2.51 & -2.47 & -3.06 & -3.41 \\
$\mathbf{V}$ & -2.33 & -2.36 & -2.38 & -2.43 & -2.26 & -2.31 & -2.17 \\
$\mathbf{C r}$ & -2.25 & -2.16 & -2.03 & -2.02 & -1.95 & -1.72 & -1.41 \\
$\mathbf{M n}$ & -1.92 & -1.7 & -1.53 & -1.57 & -1.5 & -1.29 & -1.05 \\
$\mathbf{F e}$ & -2.33 & -2.31 & -2.23 & -2.25 & -2.25 & -2.03 & -1.22 \\
$\mathbf{C o}$ & -1.96 & -1.78 & -1.64 & -1.74 & -1.72 & -1.38 & -1.09 \\
$\mathbf{N i}$ & -1.68 & -1.39 & -1.22 & -1.19 & -1.24 & -1.08 & -0.93 \\
$\mathbf{C u}$ & -1.34 & -1.13 & -1.11 & -1.17 & -1.12 & -1.01 & -0.93 \\
$\mathbf{Z n}$ & -1.3 & -1.36 & -1.36 & -1.43 & -1.36 & -1.37 & -1.41 \\
$\mathbf{Y}$ & -1.95 & -1.85 & -1.83 & -1.97 & -1.84 & -2.2 & -2.95 \\
$\mathbf{Z r}$ & -2.17 & -2.31 & -2.59 & -2.66 & -2.59 & -3 & -3.28 \\
$\mathbf{N b}$ & -2.38 & -2.81 & -3.35 & -3.22 & -3.28 & -3.61 & -3.94 \\
$\mathbf{M o}$ & -2.46 & -2.61 & -2.95 & -2.86 & -2.88 & -3.15 & -3.11 \\
$\mathbf{T c}$ & -2.17 & -2.28 & -2.48 & -2.51 & -2.28 & -2.45 & -1.94 \\
$\mathbf{R u}$ & -2.13 & -2.21 & -2.48 & -2.43 & -2.18 & -2.39 & -2.07 \\
$\mathbf{R h}$ & -1.96 & -1.88 & -2 & -1.94 & -1.76 & -1.76 & -1.29 \\
$\mathbf{P d}$ & -1.75 & -1.49 & -1.19 & -1.16 & -1.23 & -0.97 & -0.84 \\
$\mathbf{A g}$ & -1.29 & -1.18 & -1.07 & -1.06 & -1.08 & -0.97 & -1.03 \\
$\mathbf{C d}$ & -1.26 & -1.48 & -1.76 & -1.69 & -1.6 & -1.67 & -1.75 \\
$\mathbf{H f}$ & -2.55 & -2.61 & -2.85 & -2.85 & -2.85 & -3.4 & -4 \\
$\mathbf{T a}$ & -2.82 & -3.06 & -3.68 & -3.32 & -3.55 & -3.96 & -4.41 \\
$\mathbf{W}$ & -2.86 & -3.11 & -3.33 & -3.27 & -3.07 & -3.27 & -2.89 \\
$\mathbf{R e}$ & -2.61 & -2.74 & -3.03 & -2.92 & -2.76 & -3.07 & -2.68 \\
$\mathbf{O s}$ & -2.55 & -2.64 & -2.83 & -2.74 & -2.51 & -2.56 & -2.17 \\
$\mathbf{I r}$ & -2.31 & -2.19 & -2.09 & -2.07 & -1.92 & -1.75 & -1.2 \\
$\mathbf{P t}$ & -1.56 & -1.5 & -1.46 & -1.38 & -1.29 & -1.08 & -0.84 \\
$\mathbf{A u}$ & -1.23 & -1.11 & -0.92 & -0.98 & -0.98 & -0.84 & -0.7 \\
$\mathbf{H g}$ & -1.18 & -1.18 & -1.03 & -1.07 & -1.2 & -1.33 & -1.05 \\
\hline & & & & & & & \\
\hline
\end{tabular}


Table S6. Predicted adsorption energy of $\mathrm{Li}_{2} \mathrm{~S}_{5}$.

\begin{tabular}{|c|c|c|c|c|c|c|c|}
\hline & $4 \mathrm{C}$ & $3 \mathrm{C} 1 \mathrm{~N}$ & $2 \mathrm{C} 2 \mathrm{~N}-\mathrm{S}$ & $2 \mathrm{C} 2 \mathrm{~N}-\mathrm{L}$ & $2 \mathrm{C} 2 \mathrm{~N}-\mathrm{O}$ & $1 \mathrm{C} 3 \mathrm{~N}$ & $4 N$ \\
\hline Sc & -2.03 & -1.97 & -2.04 & -2.11 & -2.01 & -2.27 & -2.77 \\
\hline $\mathbf{T i}$ & -2.23 & -2.36 & -2.71 & -2.69 & -2.65 & -3.34 & -3.73 \\
\hline $\mathbf{V}$ & -2.42 & -2.45 & -2.48 & -2.53 & -2.36 & -2.41 & -2.23 \\
\hline $\mathrm{Cr}$ & -2.28 & -2.19 & -2.04 & -2.03 & -1.96 & -1.69 & -1.34 \\
\hline Mn & -1.97 & -1.75 & -1.58 & -1.61 & -1.54 & -1.29 & -1.01 \\
\hline $\mathrm{Fe}$ & -2.43 & -2.44 & -2.37 & -2.38 & -2.36 & -2.11 & -1.17 \\
\hline Co & -1.91 & -1.74 & -1.61 & -1.7 & -1.67 & -1.29 & -0.96 \\
\hline $\mathbf{N i}$ & -1.64 & -1.33 & -1.18 & -1.13 & -1.18 & -1.02 & -0.85 \\
\hline $\mathbf{C u}$ & -1.28 & -1.07 & -1.04 & -1.1 & -1.05 & -0.93 & -0.84 \\
\hline $\mathbf{Z n}$ & -1.21 & -1.27 & -1.29 & -1.36 & -1.3 & -1.3 & -1.32 \\
\hline $\mathbf{Y}$ & -1.97 & -1.84 & -1.82 & -1.96 & -1.82 & -2.25 & -3.13 \\
\hline $\mathbf{Z r}$ & -2.25 & -2.42 & -2.75 & -2.82 & -2.75 & -3.23 & -3.54 \\
\hline $\mathbf{N b}$ & -2.47 & -2.96 & -3.58 & -3.45 & -3.52 & -3.88 & -4.21 \\
\hline Mo & -2.5 & -2.68 & -3.06 & -2.96 & -2.99 & -3.3 & -3.27 \\
\hline Tc & -2.3 & -2.45 & -2.67 & -2.72 & -2.47 & -2.66 & -2.09 \\
\hline $\mathbf{R u}$ & -2.17 & -2.29 & -2.62 & -2.56 & -2.3 & -2.54 & -2.17 \\
\hline $\mathbf{R h}$ & -1.98 & -1.94 & -2.11 & -2.02 & -1.83 & -1.82 & -1.3 \\
\hline Pd & -1.71 & -1.45 & -1.14 & -1.09 & -1.16 & -0.89 & -0.73 \\
\hline Ag & -1.25 & -1.15 & -1.04 & -1.02 & -1.04 & -0.92 & -1 \\
\hline $\mathrm{Cd}$ & -1.15 & -1.41 & -1.74 & -1.64 & -1.56 & -1.65 & -1.73 \\
\hline Hf & -2.61 & -2.68 & -2.96 & -2.96 & -2.95 & -3.59 & -4.27 \\
\hline $\mathbf{T a}$ & -2.96 & -3.2 & -3.88 & -3.53 & -3.77 & -4.25 & -4.71 \\
\hline $\mathbf{W}$ & -2.95 & -3.21 & -3.43 & -3.4 & -3.16 & -3.37 & -2.97 \\
\hline $\mathbf{R e}$ & -2.82 & -2.97 & -3.31 & -3.2 & -3.04 & -3.39 & -2.96 \\
\hline Os & -2.65 & -2.76 & -2.99 & -2.89 & -2.64 & -2.69 & -2.23 \\
\hline Ir & -2.39 & -2.28 & -2.18 & -2.15 & -1.99 & -1.79 & -1.17 \\
\hline $\mathbf{P t}$ & -1.53 & -1.49 & -1.51 & -1.42 & -1.29 & -1.11 & -0.86 \\
\hline $\mathbf{A u}$ & -1.23 & -1.11 & -0.92 & -0.98 & -0.96 & -0.82 & -0.66 \\
\hline Hg & -1.04 & -1.07 & -0.92 & -0.96 & -1.11 & -1.23 & -0.9 \\
\hline
\end{tabular}


Table S7. Predicted adsorption energy of $\mathrm{Li}_{2} \mathrm{~S}_{6}$.

\begin{tabular}{cccccccc}
\hline & $\mathbf{4 C}$ & $\mathbf{3 C 1 N}$ & $\mathbf{2 C 2 N - S}$ & $\mathbf{2 C 2 N}-\mathbf{L}$ & $\mathbf{2 C 2 N}-\mathbf{O}$ & $\mathbf{1 C 3 N}$ & $\mathbf{4 N}$ \\
\hline $\mathbf{S c}$ & -1.74 & -1.7 & -1.8 & -1.88 & -1.77 & -2.11 & -2.71 \\
$\mathbf{T i}$ & -1.87 & -2.03 & -2.48 & -2.47 & -2.43 & -3.24 & -3.66 \\
$\mathbf{V}$ & -2.08 & -2.14 & -2.21 & -2.26 & -2.09 & -2.16 & -1.99 \\
$\mathbf{C r}$ & -2 & -1.93 & -1.81 & -1.79 & -1.72 & -1.46 & -1.09 \\
$\mathbf{M n}$ & -1.65 & -1.47 & -1.34 & -1.37 & -1.3 & -1.07 & -0.78 \\
$\mathbf{F e}$ & -2.16 & -2.23 & -2.19 & -2.19 & -2.15 & -1.91 & -0.92 \\
$\mathbf{C o}$ & -1.65 & -1.51 & -1.41 & -1.49 & -1.46 & -1.08 & -0.72 \\
$\mathbf{N i}$ & -1.39 & -1.11 & -0.98 & -0.93 & -0.98 & -0.84 & -0.69 \\
$\mathbf{C u}$ & -1.05 & -0.86 & -0.85 & -0.9 & -0.86 & -0.75 & -0.67 \\
$\mathbf{Z n}$ & -0.9 & -0.98 & -1.02 & -1.09 & -1.03 & -1.06 & -1.08 \\
$\mathbf{Y}$ & -1.74 & -1.56 & -1.53 & -1.69 & -1.54 & -2.05 & -3.07 \\
$\mathbf{Z r}$ & -1.99 & -2.2 & -2.61 & -2.7 & -2.63 & -3.18 & -3.5 \\
$\mathbf{N b}$ & -2.28 & -2.85 & -3.58 & -3.45 & -3.53 & -3.89 & -4.22 \\
$\mathbf{M o}$ & -2.31 & -2.53 & -2.99 & -2.89 & -2.93 & -3.28 & -3.25 \\
$\mathbf{T c}$ & -1.97 & -2.16 & -2.46 & -2.52 & -2.28 & -2.51 & -1.91 \\
$\mathbf{R u}$ & -1.83 & -2 & -2.4 & -2.35 & -2.09 & -2.37 & -1.98 \\
$\mathbf{R h}$ & -1.7 & -1.71 & -1.95 & -1.85 & -1.64 & -1.66 & -1.12 \\
$\mathbf{P d}$ & -1.39 & -1.16 & -0.85 & -0.81 & -0.88 & -0.6 & -0.45 \\
$\mathbf{A g}$ & -0.99 & -0.92 & -0.83 & -0.81 & -0.82 & -0.71 & -0.81 \\
$\mathbf{C d}$ & -0.79 & -1.08 & -1.45 & -1.35 & -1.26 & -1.4 & -1.51 \\
$\mathbf{H f}$ & -2.43 & -2.51 & -2.83 & -2.85 & -2.84 & -3.58 & -4.27 \\
$\mathbf{T a}$ & -2.86 & -3.11 & -3.86 & -3.52 & -3.76 & -4.27 & -4.74 \\
$\mathbf{W}$ & -2.75 & -3.06 & -3.31 & -3.3 & -3.05 & -3.28 & -2.86 \\
$\mathbf{R e}$ & -2.58 & -2.75 & -3.16 & -3.06 & -2.9 & -3.3 & -2.85 \\
$\mathbf{O s}$ & -2.35 & -2.5 & -2.77 & -2.68 & -2.43 & -2.5 & -2.01 \\
$\mathbf{I r}$ & -2.16 & -2.07 & -2 & -1.97 & -1.8 & -1.6 & -0.95 \\
$\mathbf{P t}$ & -1.22 & -1.23 & -1.32 & -1.22 & -1.09 & -0.94 & -0.66 \\
$\mathbf{A u}$ & -1.01 & -0.91 & -0.73 & -0.79 & -0.78 & -0.63 & -0.48 \\
$\mathbf{H g}$ & -0.73 & -0.79 & -0.68 & -0.72 & -0.86 & -1 & -0.66 \\
\hline & & & & & & & \\
\hline
\end{tabular}


Table S8. Predicted adsorption energy of $\mathrm{Li}_{2} \mathrm{~S}_{7}$.

\begin{tabular}{cccccccc}
\hline & $\mathbf{4 C}$ & $\mathbf{3 C 1 N}$ & $\mathbf{2 C 2 N}-\mathbf{S}$ & $\mathbf{2 C 2 N}-\mathbf{L}$ & $\mathbf{2 C 2 N}-\mathbf{O}$ & $\mathbf{1 C 3 N}$ & $\mathbf{4 N}$ \\
\hline $\mathbf{S c}$ & -1.81 & -1.74 & -1.79 & -1.86 & -1.76 & -2 & -2.45 \\
$\mathbf{T i}$ & -1.96 & -2.06 & -2.38 & -2.37 & -2.33 & -2.95 & -3.31 \\
$\mathbf{V}$ & -2.1 & -2.14 & -2.17 & -2.22 & -2.06 & -2.12 & -1.98 \\
$\mathbf{C r}$ & -2.02 & -1.94 & -1.82 & -1.82 & -1.75 & -1.53 & -1.24 \\
$\mathbf{M n}$ & -1.73 & -1.53 & -1.39 & -1.43 & -1.35 & -1.16 & -0.92 \\
$\mathbf{F e}$ & -2.15 & -2.14 & -2.08 & -2.09 & -2.09 & -1.87 & -1.08 \\
$\mathbf{C o}$ & -1.72 & -1.56 & -1.44 & -1.53 & -1.52 & -1.19 & -0.93 \\
$\mathbf{N i}$ & -1.44 & -1.15 & -1.02 & -0.98 & -1.03 & -0.9 & -0.76 \\
$\mathbf{C u}$ & -1.16 & -0.96 & -0.94 & -1 & -0.96 & -0.85 & -0.77 \\
$\mathbf{Z n}$ & -1.11 & -1.17 & -1.19 & -1.25 & -1.19 & -1.2 & -1.25 \\
$\mathbf{Y}$ & -1.74 & -1.61 & -1.6 & -1.73 & -1.6 & -1.99 & -2.79 \\
$\mathbf{Z r}$ & -1.99 & -2.14 & -2.45 & -2.52 & -2.46 & -2.89 & -3.18 \\
$\mathbf{N b}$ & -2.15 & -2.6 & -3.17 & -3.05 & -3.11 & -3.46 & -3.77 \\
$\mathbf{M o}$ & -2.22 & -2.38 & -2.72 & -2.64 & -2.66 & -2.95 & -2.92 \\
$\mathbf{T c}$ & -1.99 & -2.12 & -2.34 & -2.38 & -2.15 & -2.33 & -1.84 \\
$\mathbf{R u}$ & -1.89 & -1.99 & -2.28 & -2.23 & -1.99 & -2.22 & -1.9 \\
$\mathbf{R h}$ & -1.75 & -1.69 & -1.84 & -1.76 & -1.6 & -1.6 & -1.14 \\
$\mathbf{P d}$ & -1.56 & -1.32 & -1.04 & -1.01 & -1.07 & -0.83 & -0.7 \\
$\mathbf{A g}$ & -1.13 & -1.03 & -0.94 & -0.93 & -0.95 & -0.85 & -0.91 \\
$\mathbf{C d}$ & -1.06 & -1.28 & -1.58 & -1.49 & -1.41 & -1.5 & -1.58 \\
$\mathbf{H f}$ & -2.32 & -2.39 & -2.65 & -2.66 & -2.65 & -3.23 & -3.87 \\
$\mathbf{T a}$ & -2.62 & -2.85 & -3.49 & -3.16 & -3.38 & -3.83 & -4.26 \\
$\mathbf{W}$ & -2.62 & -2.87 & -3.09 & -3.04 & -2.84 & -3.05 & -2.69 \\
$\mathbf{R e}$ & -2.44 & -2.58 & -2.9 & -2.8 & -2.65 & -2.98 & -2.6 \\
$\mathbf{O s}$ & -2.33 & -2.43 & -2.64 & -2.55 & -2.33 & -2.39 & -2 \\
$\mathbf{I r}$ & -2.11 & -2 & -1.91 & -1.89 & -1.75 & -1.59 & -1.05 \\
$\mathbf{P t}$ & -1.37 & -1.33 & -1.34 & -1.26 & -1.17 & -0.99 & -0.77 \\
$\mathbf{A u}$ & -1.1 & -1 & -0.83 & -0.89 & -0.88 & -0.75 & -0.62 \\
$\mathbf{H g}$ & -0.99 & -1.01 & -0.89 & -0.93 & -1.04 & -1.19 & -0.91 \\
\hline & & & & & & & \\
\hline
\end{tabular}


Table S9. Predicted adsorption energy of $\mathrm{Li}_{2} \mathrm{~S}_{8}$.

\begin{tabular}{|c|c|c|c|c|c|c|c|}
\hline & $4 \mathrm{C}$ & $3 \mathrm{C} 1 \mathrm{~N}$ & $2 \mathrm{C} 2 \mathrm{~N}-\mathrm{S}$ & $2 \mathrm{C} 2 \mathrm{~N}-\mathrm{L}$ & $2 \mathrm{C} 2 \mathrm{~N}-\mathrm{O}$ & $1 \mathrm{C} 3 \mathrm{~N}$ & $4 N$ \\
\hline Sc & -1.67 & -1.62 & -1.68 & -1.75 & -1.65 & -1.92 & -2.42 \\
\hline $\mathbf{T i}$ & -1.86 & -1.99 & -2.35 & -2.33 & -2.29 & -2.98 & -3.38 \\
\hline $\mathbf{V}$ & -2.07 & -2.1 & -2.13 & -2.19 & -2.02 & -2.07 & -1.9 \\
\hline $\mathrm{Cr}$ & -1.94 & -1.85 & -1.72 & -1.71 & -1.64 & -1.39 & -1.06 \\
\hline Mn & -1.62 & -1.41 & -1.26 & -1.3 & -1.22 & -1 & -0.74 \\
\hline $\mathrm{Fe}$ & -2.08 & -2.1 & -2.04 & -2.05 & -2.03 & -1.8 & -0.91 \\
\hline Co & -1.58 & -1.42 & -1.3 & -1.38 & -1.36 & -1.01 & -0.7 \\
\hline $\mathbf{N i}$ & -1.28 & -0.99 & -0.85 & -0.81 & -0.86 & -0.72 & -0.57 \\
\hline $\mathrm{Cu}$ & -0.99 & -0.8 & -0.77 & -0.83 & -0.78 & -0.67 & -0.59 \\
\hline $\mathbf{Z n}$ & -0.91 & -0.98 & -1 & -1.07 & -1.01 & -1.02 & -1.05 \\
\hline $\mathbf{Y}$ & -1.64 & -1.49 & -1.47 & -1.61 & -1.47 & -1.9 & -2.79 \\
\hline $\mathbf{Z r}$ & -1.88 & -2.05 & -2.37 & -2.45 & -2.38 & -2.86 & -3.19 \\
\hline $\mathbf{N b}$ & -2.07 & -2.56 & -3.19 & -3.07 & -3.14 & -3.52 & -3.84 \\
\hline Mo & -2.14 & -2.32 & -2.69 & -2.59 & -2.63 & -2.95 & -2.94 \\
\hline Tc & -1.93 & -2.08 & -2.3 & -2.35 & -2.12 & -2.3 & -1.76 \\
\hline $\mathbf{R u}$ & -1.82 & -1.94 & -2.27 & -2.22 & -1.97 & -2.21 & -1.85 \\
\hline $\mathbf{R h}$ & -1.64 & -1.6 & -1.77 & -1.68 & -1.5 & -1.5 & -1.01 \\
\hline Pd & -1.39 & -1.14 & -0.85 & -0.81 & -0.87 & -0.61 & -0.47 \\
\hline Ag & -0.96 & -0.86 & -0.76 & -0.75 & -0.76 & -0.66 & -0.73 \\
\hline Cd & -0.85 & -1.11 & -1.43 & -1.34 & -1.25 & -1.35 & -1.43 \\
\hline Hf & -2.24 & -2.31 & -2.59 & -2.6 & -2.59 & -3.24 & -3.93 \\
\hline Ta & -2.58 & -2.81 & -3.5 & -3.17 & -3.39 & -3.9 & -4.36 \\
\hline $\mathbf{W}$ & -2.59 & -2.85 & -3.07 & -3.05 & -2.82 & -3.04 & -2.66 \\
\hline $\mathbf{R e}$ & -2.48 & -2.63 & -2.97 & -2.87 & -2.71 & -3.07 & -2.67 \\
\hline Os & -2.31 & -2.42 & -2.65 & -2.56 & -2.33 & -2.38 & -1.94 \\
\hline Ir & -2.05 & -1.95 & -1.85 & -1.83 & -1.68 & -1.48 & -0.9 \\
\hline $\mathbf{P t}$ & -1.19 & -1.17 & -1.2 & -1.12 & -1 & -0.83 & -0.61 \\
\hline $\mathbf{A u}$ & -0.95 & -0.84 & -0.66 & -0.72 & -0.71 & -0.58 & -0.43 \\
\hline Hg & -0.77 & -0.81 & -0.68 & -0.73 & -0.85 & -0.99 & -0.67 \\
\hline
\end{tabular}




\section{References}

1. Kresse, G.; Hafner, J., Ab initio molecular dynamics for liquid metals. Phys. Rev. B 1993, 47, 558-561.

2. Kresse, G.; Furthmuller, J., Efficiency of ab-initio total energy calculations for metals and semiconductors using a plane-wave basis set. Comp. Mater. Sci. 1996, 6, 15-50.

3. Kresse, G.; Furthmuller, J., Efficient iterative schemes for ab initio total-energy calculations using a plane-wave basis set. Phys. Rev. B 1996, 54, 11169-11186.

4. Blochl, P. E., Projector augmented-wave method. Phys. Rev. B 1994, 50, 17953-17979.

5. Kresse, G.; Joubert, D., From ultrasoft pseudopotentials to the projector augmented-wave method. Phys. Rev. B 1999, 59, 1758-1775.

6. Hammer, B.; Hansen, L. B.; Nørskov, J. K., Improved adsorption energetics within density-functional theory using revised Perdew-Burke-Ernzerhof functionals. Phys. Rev. B 1999, 59, 7413-7421.

7. Grimme, S.; Antony, J.; Ehrlich, S.; Krieg, H., A consistent and accurate ab initio parametrization of density functional dispersion correction (DFT-D) for the 94 elements H-Pu. $J$ Chem. Phys. 2010, $132,154104$.

8. Bahn, S. R.; Jacobsen, K. W., An object-oriented scripting interface to a legacy electronic structure code. Comput. Sci. Eng. 2002, 4, 56-66.

9. Tang, W.; Sanville, E.; Henkelman, G., A grid-based Bader analysis algorithm without lattice bias. J. Phys.: Condens. Matter 2009, 21, 084204.

10. Manz, T. A.; Limas, N. G., Introducing DDEC6 atomic population analysis: part 1. Charge partitioning theory and methodology. RSC Adv. 2016, 6, 47771-47801.

11. Jmol: an open-source Java viewer for chemical structures in 3D. http://www.jmol.org/.

12. Xie, T.; Grossman, J. C., Crystal Graph Convolutional Neural Networks for an Accurate and Interpretable Prediction of Material Properties. Phys. Rev. Lett. 2018, 120, 145301.

13. Imbalanced Dataset Sampler. https://github.com/ufoym/imbalanced-dataset-sampler.

14. Peng, L.; Wei, Z.; Wan, C.; Li, J.; Chen, Z.; Zhu, D.; Baumann, D.; Liu, H.; Allen, C. S.; Xu, X.; Kirkland, A. I.; Shakir, I.; Almutairi, Z.; Tolbert, S.; Dunn, B.; Huang, Y.; Sautet, P.; Duan, X., A fundamental look at electrocatalytic sulfur reduction reaction. Nat. Catal. 2020, 3, 762-770.

15. Nørskov, J. K.; Rossmeisl, J.; Logadottir, A.; Lindqvist, L.; Kitchin, J. R.; Bligaard, T.; Jónsson, H., Origin of the Overpotential for Oxygen Reduction at a Fuel-Cell Cathode. J. Phys. Chem. B 2004, $108,17886-17892$. 